\title{
STUDIES IN STEROID METABOLISM. XXII. THE ADRENAL RESPONSE TO ACTH IN YOUNG AND OLD MEN ${ }^{1}$
}

\author{
BY KONRAD DOBRINER 2 \\ The data were assembled and the manuscript prepared by: ${ }^{3}$ \\ ATTALLAH KAPPAS, ${ }^{4}$ C. P. RHOADS, AND T. F. GALLAGHER
}

(From the Sloan-Kettering Institute for Cancer Research, New York, N. Y.)

(Submitted for publication August 3, 1953; accepted September 16, 1953)

Recent data based on isolation and quantitation of metabolites of adrenal hormones indicated that adrenal function was independent of chronological age (1). It was important, therefore, to study the capacity of older men to respond to adrenal stimulation and to compare the character of this response with that of younger men, since a prime characteristic of the adrenal gland is the capacity for quick and often large scale response. In a previous study based on the adrenal response to intramuscularly administered ACTH, Solomon and Shock (2) concluded that the ability of the adrenal cortex to secrete hormones of the 11, 17oxygenated type was not grossly impaired in the aged. Pincus (3) was unable to detect a significant difference in adrenal responsiveness to ACTH between healthy young men and old men.

The results obtained in this study, in which the individual metabolic products of adrenal cortical hormones were separated and identified, confirm and extend these general conclusions.

\section{METHODS}

Four normal male subjects were studied: the ages were $31,32,63$, and 65 years. All lived on a metabolic ward during the course of the study, and metabolic balance data on these men have been reported (4). The essential details of the study are as follows: a control pe-

1 The authors gratefully acknowledge the assistance of grants from the American Cancer Society (on recommendation of the Committee on Growth of the National Research Council), the Commonwealth Fund, the Anna Fuller Fund, the Lillia Babbitt Hyde Foundation, and the National Cancer Institute of the National Institutes of Health of the United States Public Health Service.

2 Konrad Dobriner died March 10, 1952.

3 So far as we are aware, the interpretation of the data and the conclusions drawn are in essential agreement with the views held by the late Dr. Dobriner.

4 Research Fellow of the American Cancer Society upon recommendation of the Committee on Growth of the National Research Council. riod, lasting 15 days was immediately followed by four consecutive periods, each of 3 days duration, during which $100 \mathrm{mg}$. of ACTH was given intramuscularly daily in four divided doses. Two weeks after the end of the last ACTH period, another control period lasting 6 days was obtained. The urinary steroids were examined during these periods by methods detailed in previous reports $(1,5)$. All urines were hydrolyzed according to Method C, which has been described in detail (1). In brief, this method employed acidification of the urine to pH 1 and continuous ether extraction for 48 hours. This ether extract was washed with alkali and the washes, after adjustment to $\mathrm{pH} 5$, were incubated at $37^{\circ}$ with beef liver $\beta$-glucuronidase for 48 hours. The digest was then brought to $\mathrm{pH} 1$ and extracted with ether. A neutral fraction was obtained from this extract as described. The spent urine from the $\mathrm{pH} 1$ extraction, the alkali soluble fraction obtained after enzymatic hydrolysis, as well as the extracted enzyme hydrolysate were hydrolyzed with boiling acid and re-extracted with ether. The extracts obtained after mild hydrolysis ( $\mathrm{pH} 1$, enzyme) were worked up separately from those obtained after hot acid hydrolysis. Variable amounts of $\Delta^{v-11}$ etiocholenolone and $\Delta^{q-11}$ androstenolone were isolated from vigorously hydrolyzed extracts. These amounts are included in the final values for the 11-hydroxylated precursor. Non-specific chromogenic material undoubtedly accounts for the discrepancy between the values for the sum of isolated $3 \alpha$ and $3 \beta$ 17ketosteroids and the values for the original crude extracts. Chromatographic separation of water extracts, obtained after benzene-water partition according to the method of Mason (6), was done in only two subjects, $\mathrm{K} 31$ and R32.

\section{RESULTS}

\section{Subject $K 31$}

Subject K31 was a 31 year old negro male. Except for residual muscle atrophy in the lower extremities resulting from old poliomyelitis; he appeared in excellent health. Blood pressure was $120 / 70 \mathrm{~mm}$. Hg, height was 5 feet 4 inches, and weight was 158 pounds. BMR was -15 per cent. $C_{\text {in }}$ was 84 cc., $C_{p a h}$ was 397 cc. and $T_{m g}$ was $260 \mathrm{mg}$. per minute per 1.73 square meters. The reason for these low values was not apparent. 


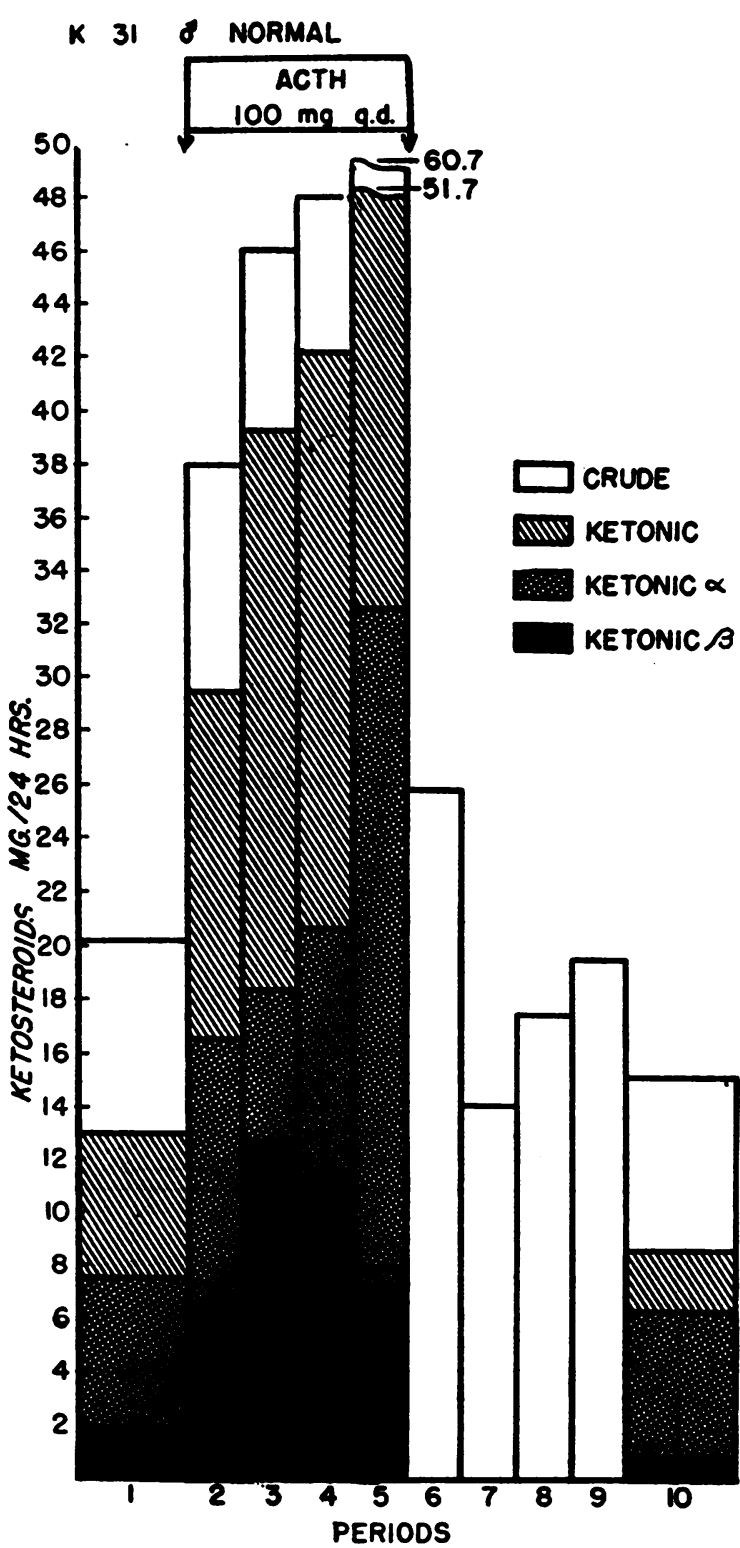

Fig. 1. Subject K31-Urinary Steroids before, durING, AND AFTer ACTH Administration

On the tenth day of ACTH administration (Period 5 ), he developed fever and a cough productive of rusty sputum containing type 9 pneumococci. Chest X-ray revealed an area of infiltration in the left lower lung field. No treatment was given. He became afebrile in one day and his lung infiltration disappeared in 4 days.

During ACTH administration there was a stepwise increase in the urinary steroids as shown in Figure 1. The $\beta$-ketonic fraction from this sub- ject represented a very high proportion (20.2 per cent) of the total $\alpha$ and $\beta$-ketosteroids during the control period; during ACTH administration this $\beta$-ketosteroid fraction increased to 41 per cent of this total. Thereafter, despite continued ACTH administration, the $\beta$-ketosteroids diminished both relatively and absolutely.

The sum of the $\mathrm{C}_{10}-11$ desoxy metabolites isolated from the $\alpha$-ketonic fraction was increased two-fold, largely as the result of greater production of etiocholanolone; the level of androsterone was not changed significantly (Figure 2 ).

K 31 \& NORMAL
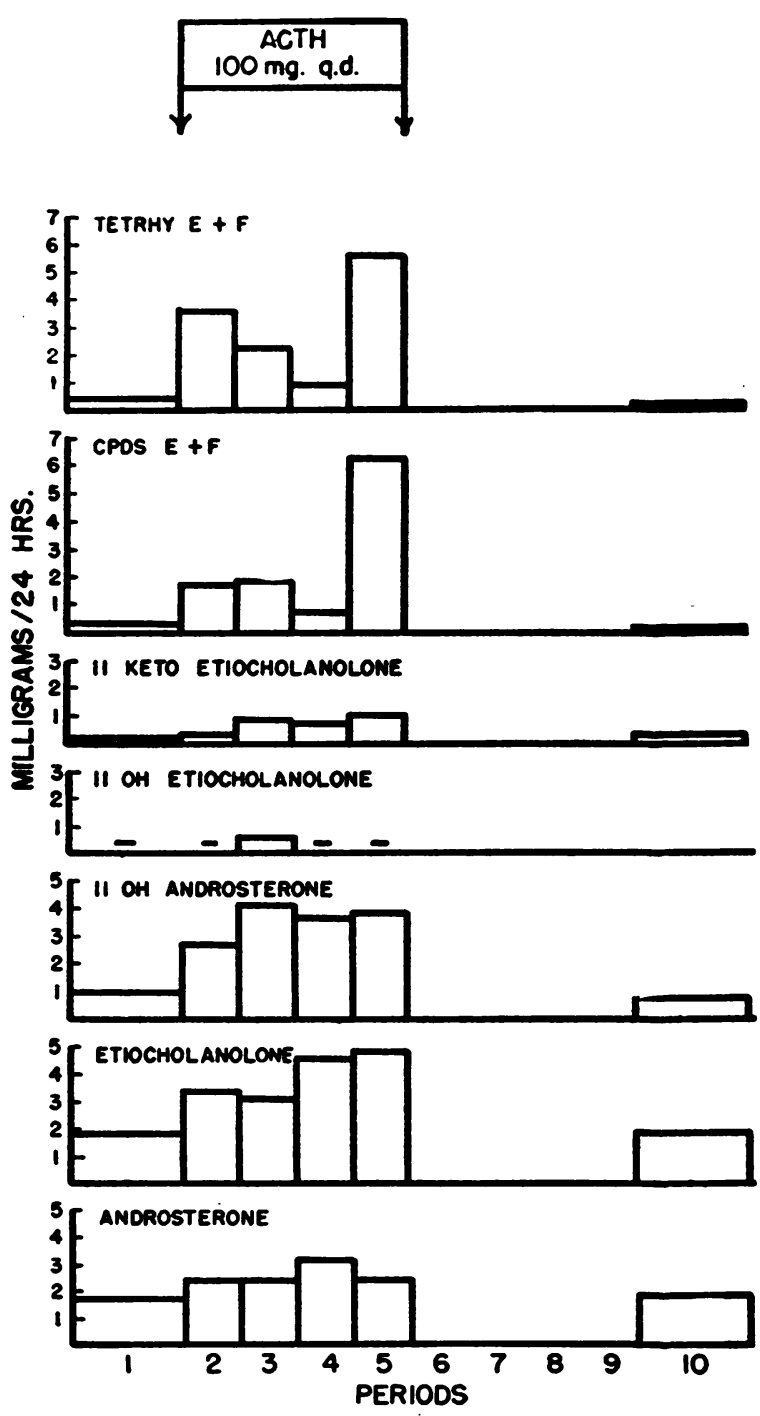

Fig. 2. Subject K31-Sterold Excretion Pattern beFORE, DURING, AND AFTER ACTH ADMINISTRation 


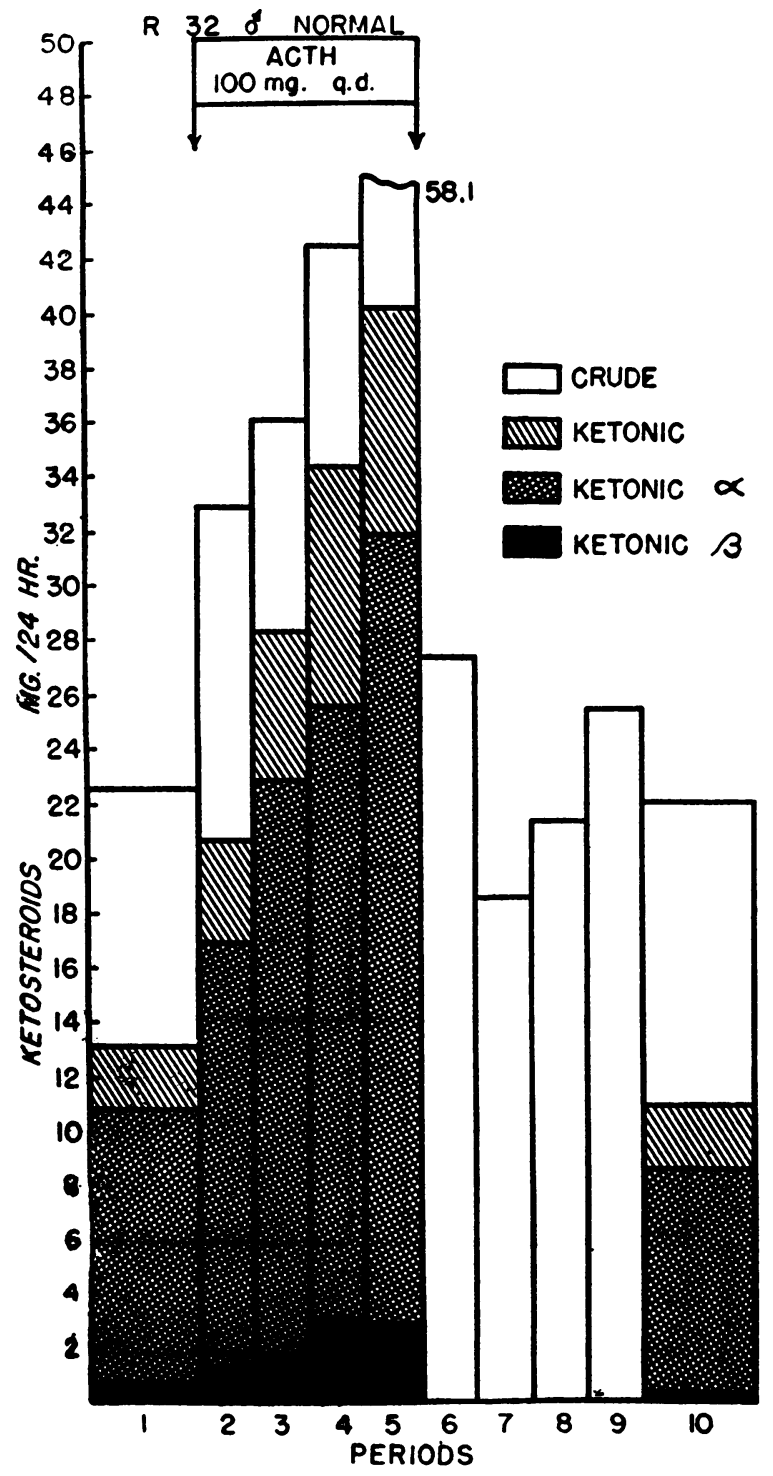

Fig. 3. SUbject R32-Urinary Steroids before, DURing, AND AFTER ACTH Administration

The increase in output of steroids of the $C_{19}-11$ oxygenated group reached a maximum of fourfold over control levels in Period 5. In this subject, the major component of this group in all periods was 11-hydroxyandrosterone, and during ACTH administration the increase in the amount of this metabolite was prompt and sustained. The amount of 11-ketoetiocholanolone increased steadily. This subject failed to excrete any significant amount of 11-hydroxyetiocholanolone. The unusually large $\beta$-ketosteroid fraction from Period 3 in this subject was chromatographed on silica gel columns and the fractions studied by infrared spec- troscopy. Dehydroisoandrosterone and isoandrosterone accounted for practically the whole weight of this material. Other investigators $(7,8)$ have demonstrated by colorimetric analyses of urinary extracts an increase in dehydroisoandrosteronelike material after ACTH stimulation.

The pattern of excretion of compounds $E$ and $\mathrm{F}$ and their "tetrahydro" derivatives was quite unusual in Periods 3 and 4. Poor chromatographic separation of these compounds from non-steroidal contaminants of the water extract is a plausible reason for the unexpected decreases noted in these periods.

\section{Subject $R 32$}

Subject R32 was a 32 year old white male in excellent health. Blood pressure was $96 / 56 \mathrm{~mm}$. $\mathrm{Hg}$, height was 5 feet $3 \frac{1}{2}$ inches, and weight was 117 pounds. Laboratory studies including blood
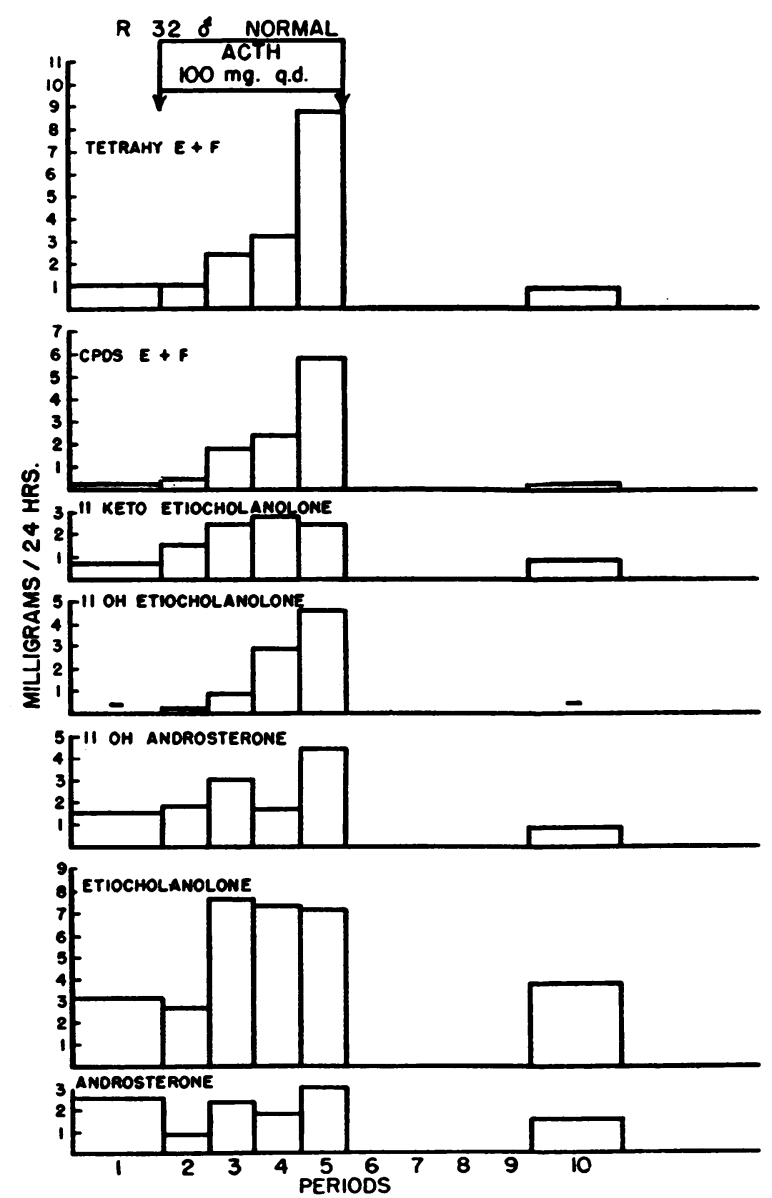

Fig. 4. Subject R32-Steroid Excretion Pattern beFORE, DURING, AND AFTER ACTH ADMINISTRATION 
counts, ESR, urinalysis, and liver and kidney function tests were all normal.

Six periods were studied in this subject; control periods before and after ACTH administration, and four 3 day periods on ACTH. Steroid excretion data are shown in Figure 3 . In this subject the administration of $\mathrm{ACTH}$ resulted in a prompt and stepwise increase in urinary ketosteroids. The $\beta$-ketonic fraction followed this same pattern of response. Twelve days after the last dose of ACTH (Period 10), the urinary steroids were at control levels.

The output of $\mathrm{C}_{19}-11$ desoxy steroids was increased almost two-fold, solely as a result of a pronounced rise in the excretion of etiocholanolone (Figure 4). The output of androsterone was essentially unchanged. The $\mathrm{C}_{19}-11$ oxygenated compounds were augmented to more than five times the control level. The most striking alteration was observed with 11-hydroxyetiocholanolone, a compound that was not found in either control period. The amount of this metabolite increased strikingly with each period, and during the final 3 days of ACTH this substance was excreted in greater amount than either of the two other major components of the $\mathrm{C}_{19}-11$ oxygenated group. The excretion of 11-hydroxyandrosterone increased irregularly but significantly. The excretion of compounds $E$ and $F$ and their "tetrahydro" derivatives increased markedly with continued adminis-

R 32 NORMAL

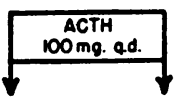

* $C_{19}$ - -11 DESOXY STEROIDS EXCRETED AS ETHOCHOLANE DERIVATIVES (RAGS A:B-CiS)

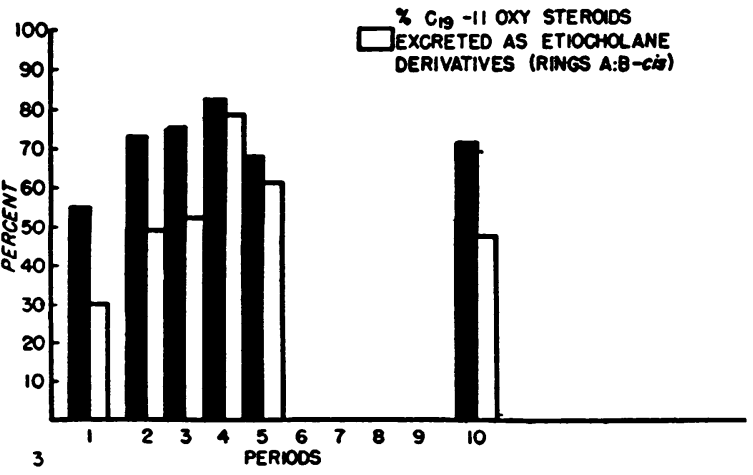

Fig. 5. SUBJECt R32-STEREOCHEMISTRY OF URINARY Ketosteroids BEFore, DURING, AND AFTER ACTH ADMINISTRATION

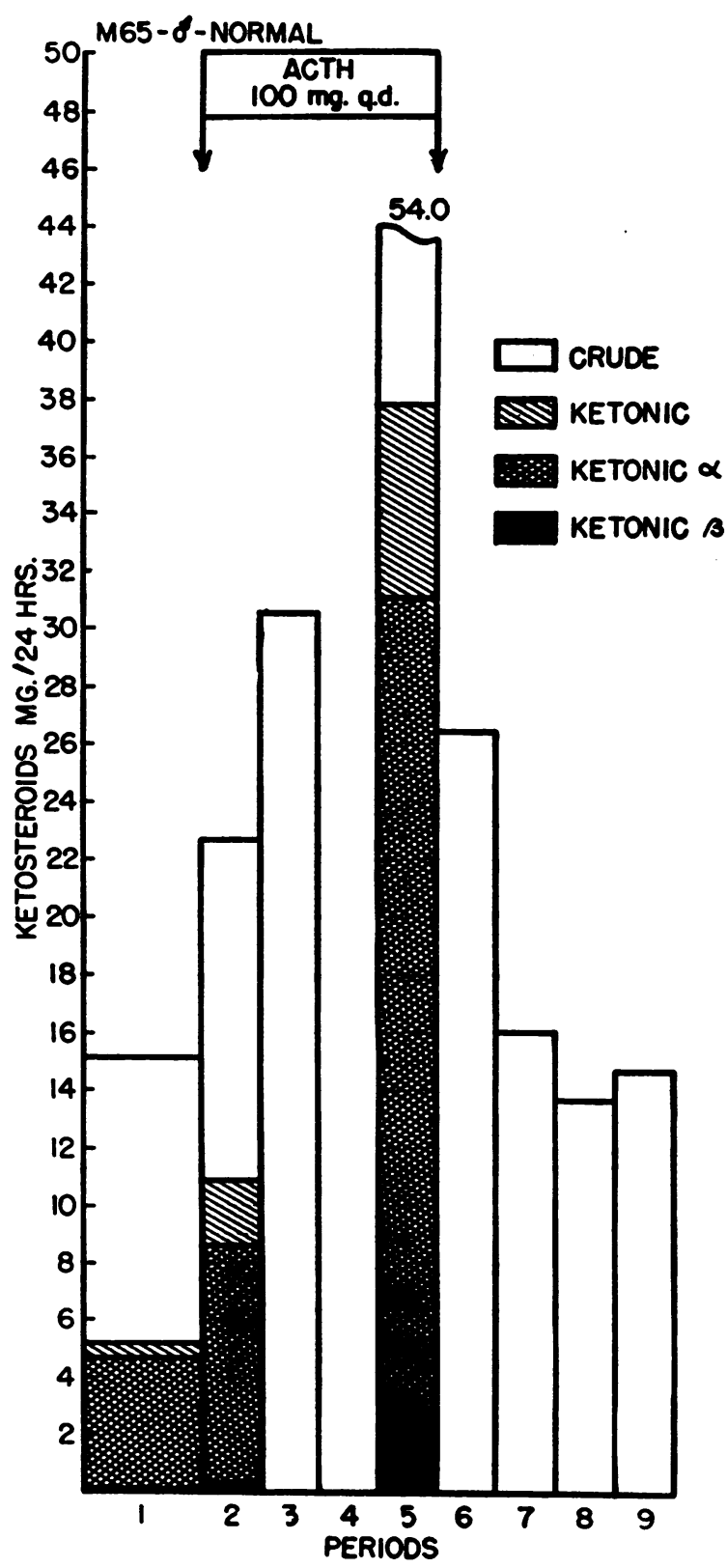

Fig. 6. Subject M65-Urinary Steroids before, durING, AND AFTER ACTH Administration

tration of ACTH, as expected. Figure 5 depicts the per cent of each of the two major groups of compounds $\left(\mathrm{C}_{19}-11\right.$ oxy, $\mathrm{C}_{19}-11$ desoxy) excreted in the form of derivatives of the parent hydrocarbon, etiocholane. This percentage was greater during the ACTH periods.

\section{Subject M65}

Subject M65 was a 65 year old white male with a past history of good health. Blood pressure was 


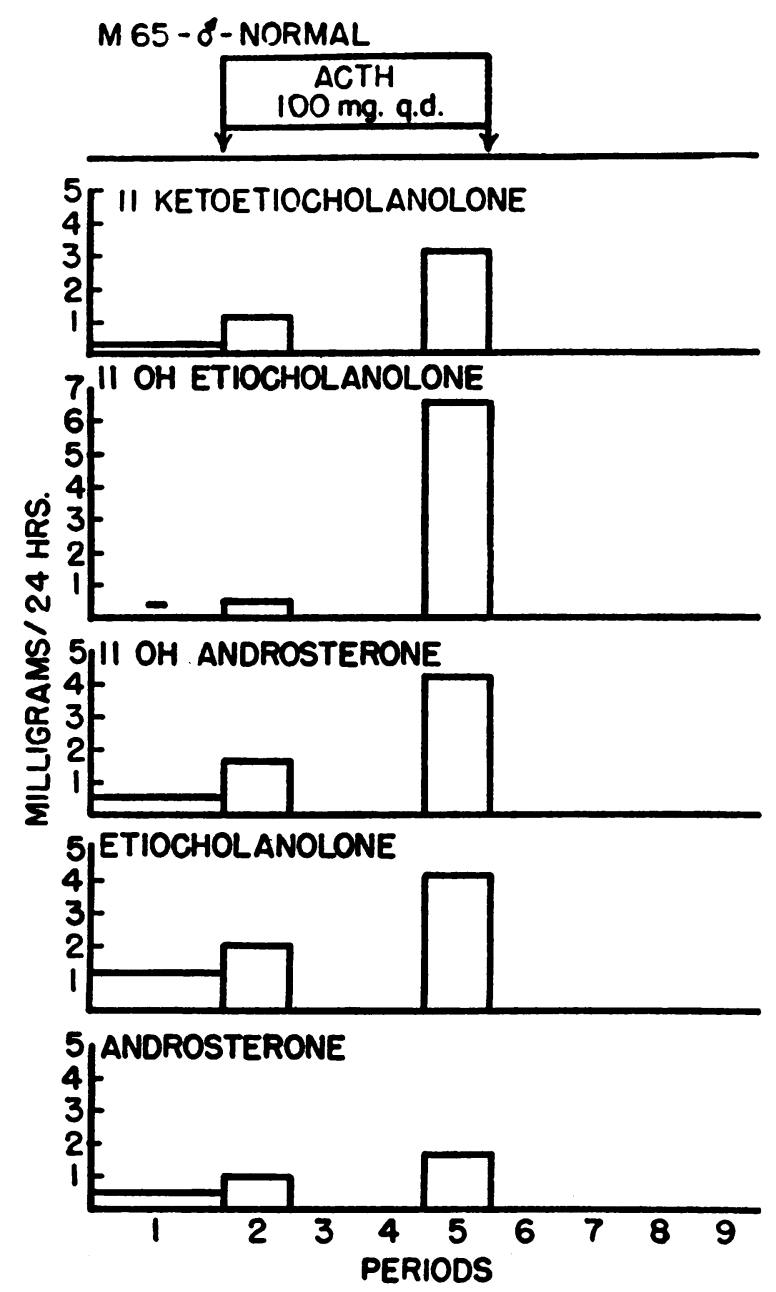

Fig. 7. Subject M65-Steroid Excretion Pattern beFORE AND DURING ACTH ADMINISTRation

$120 / 70 \mathrm{~mm} . \mathrm{Hg}$, height was 5 feet $3 \frac{1}{2}$ inches and weight was 127 pounds. He was slightly emphysematous; serologic test for syphilis was positive; ESR was 22 to $28 \mathrm{~mm}$. per hour. The reason for this increase was not evident. Liver and kidney function tests were normal. Only three periods were studied on this subject; the control period preceding ACTH therapy, and Periods 2 (days 1 to 3 on ACTH) and 5 (days 10 to 12 on ACTH). Crude ketosteroids were determined in Periods 3, 6, 7, 8 and 9 but these were not further fractionated.

This subject exhibited a prompt and marked increase in urinary steroids during $\mathrm{ACTH}$, in all essential details similar to that seen in the two younger subjects (Figure 6).

The $\mathrm{C}_{19}-11$ desoxy group in this subject while on $\mathrm{ACTH}$ increased approximately four-fold over the control level. Etiocholanolone was markedly augmented (Figure 7 ), but there was an increase in the amount of androsterone excreted as well. The $\mathrm{C}_{19}-11$ oxygenated steroids increased more than 17 times over the control level during the final 3 days of ACTH, by far the greatest absolute and proportionate increase of all the subjects. It is evident that initially there was a prompt increase in all three major components of the $\mathrm{C}_{19^{-}}$11 oxygenated group, 11-hydroxyetiocholanolone, 11-hydroxyandrosterone, and 11-ketoeticholanolone. In the final ACTH period, however, 11-hydroxyetiocholanolone represented nearly one-half of the total output of $\mathrm{C}_{19}-11$ oxygenated compounds.

The percentage of $\mathrm{C}_{19}-11$ desoxy steroids excreted in the form of etiocholane derivatives remained the same in both the control and the ACTH periods despite the several-fold increase in the total amount excreted (Figure 8). The per cent of $\mathrm{C}_{18}-11$ oxygenated compounds excreted in the form of etiocholane derivatives increased promptly and in the final period was almost twice that of the control period.

\section{Subject R63}

Subject R63 was a 63 year old white male whose general health had been excellent. Blood pressure was $94 / 58 \mathrm{~mm}$. $\mathrm{Hg}$, height was 5 feet $51 / 2$ inches and weight was 134 pounds. Laboratory and clinical examination was essentially normal.

Five periods were studied in this subject; the

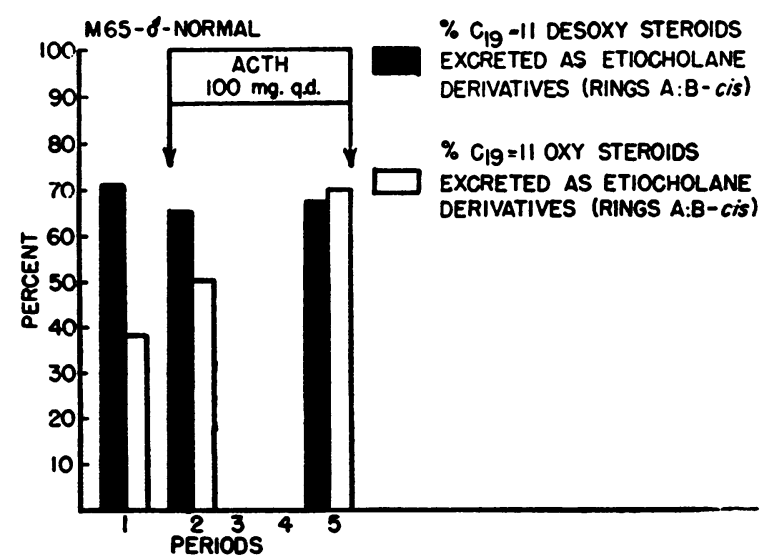

Fig. 8. SUbJect M65-STEREochemistry of Urinary Ketosteroids before and dURING ACTH AdministraTION 
R 63 \& -NORMAL

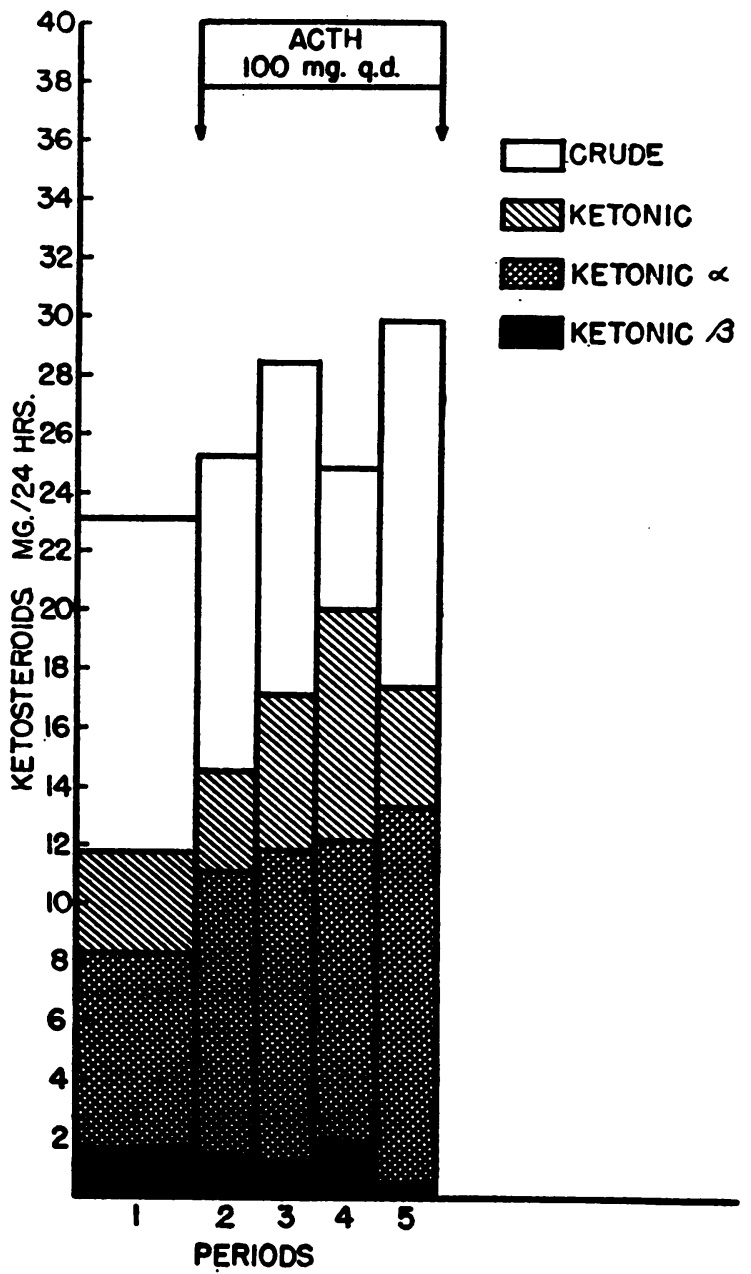

Fig. 9. Subject R63-Urinary Steroids before and DURING ACTH ADMinistration

control period preceding ACTH therapy, and the four 3 day periods on ACTH. Administration of ACTH in this subject resulted in a consistent stepwise increase in urinary steroids, but the response was smaller than with the other three subjects. There was no significant alteration in the $\beta$-ketosteroid fraction (Figure 9).

The $\mathrm{C}_{19}-11$ desoxy metabolites increased slightly during this course of ACTH, solely as the result of a slight rise in the excretion of etiocholanolone; the androsterone level was essentially unchanged (Figure 10). The $\mathrm{C}_{19}-11$ oxygenated group of compounds definitely increased during ACTH administration, but the stepwise increase observed in the other subjects was not found in this man. There was a prompt increase in excretion of 11- hydroxyandrosterone and 11-ketoetiocholanolone during the first period on ACTH, but surprisingly, as the experiment continued, these two substances diminished in amount until the final 3 day period when an increase was again evident. The fact that there was a consistent increase in the excretion of 11-hydroxyetiocholanolone makes it appear im-

\section{R 63 \&-NORMAL}
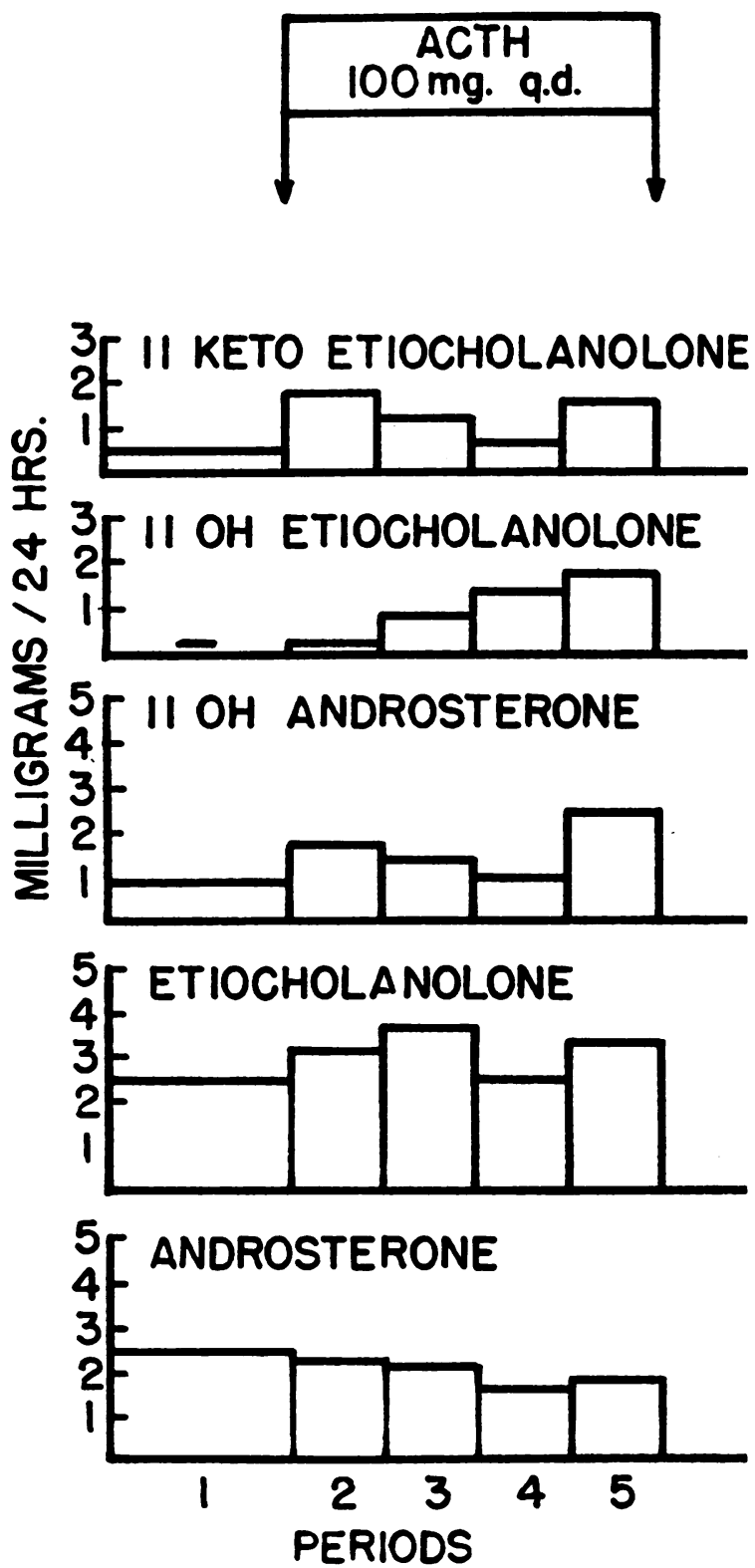

Fig. 10. Subject R63-Steroid Excretion Pattern beFORE AND DURING ACTH ADMINISTRATION 


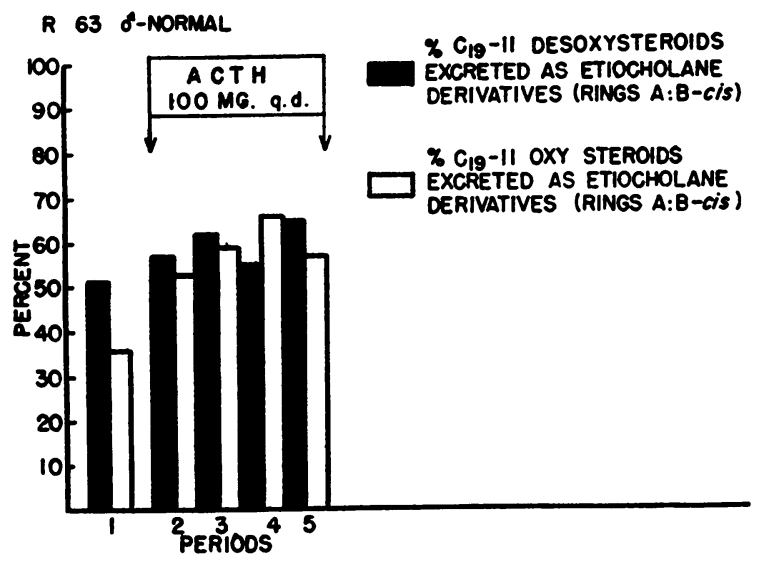

Fig. 11. SubJect R63-Stereochemistry of URINARY Ketosteroids before and dURING ACTH AdministraTION

probable that either incomplete urine samples or technical errors in separation and isolation were responsible for the unexpected decrease of the other $\mathrm{C}_{19}-11$ oxygenated steroids.

As observed with the other subjects, ACTH resulted in an immediate and sustained increase in the per cent of metabolites excreted with the normal (etiocholane) configuration at carbon 5 (Figure 11).

\section{DISCUSSION}

The adrenal response to continued daily intramuscular administration of $\mathrm{ACTH}$, as measured by urinary steroid excretion, is in the most general terms characterized by a prompt stepwise increase in neutral $\alpha$ and $\beta$-ketonic steroid fractions. When these fractions are subjected to finer analytic techniques, it is clear that the 11-oxygenated steroids are increased to a relatively greater extent than their 11-desoxy counterparts. The 11-oxygenated group may in fact become the largest portion of the $\alpha$-ketonic fraction. Both major components of the 11-oxygenated group, 11-ketoetiocholanolone and 11-hydroxyandrosterone share in this increase. In addition, 11-hydroxyetiocholanolone which was not found in the control periods of any of these subjects, appeared in substantial amounts in three out of four of them during ACTH administration. This result is interesting, especially when consideration is given to the virtual absence of this compound in the urine of a series of normal subjects (1). The major $C_{21}$ steroids identified in urine after ACTH were the 11-oxygenated "tetra- hydro" derivatives of cortisone and hydrocortisone. There was, however, spectroscopic evidence that a large number of steroids of unknown structure appeared in the urine in trace amounts after ACTH administration. Few of these trace substances have been chemically identified as yet.

The $\mathrm{C}_{19}-11$ desoxy steroids of the $\alpha$-ketonic fraction are increased during ACTH administration although the rise is relatively not as great as that undergone by the 11-oxygenated group of steroids. One compound, etiocholanolone, was responsible for most of this increase although androsterone was often excreted in larger amounts than during control periods. The precursors of these $\mathrm{C}_{19}-11$ desoxy metabolites appearing after ACTH stimulation are not known with certainty at present. It is significant, however, that both $17 \alpha$ hydroxy progesterone and "Reichsteins Substance S" (17 $\alpha, 21$-dihydroxy- $\Delta^{4}$-pregnene-3, 20-dione) have been demonstrated to be precursors of the 17 ketosteroids etiocholanolone and androsterone by isotopic experiments from this laboratory $(9,10)$.

Although several common features of this adrenal response to ACTH are shared by all four subjects, there are marked individual variations in response. It is clear that these individual differences are not characteristic of age. From these data, it is impossible to distinguish any difference in response to ACTH of "young" as compared with "old" subjects. This amply confirms the similar conclusions reached by other investigators (4) after an analysis of the metabolic balance data on these subjects.

It does seem clear from this study that the response to ACTH varies quantitatively with different individuals. That these variations may be specific for the individual and dependent to a large degree on such constitutional factors as glandular size, muscle mass or genetically determined metabolic factors is suggested by the high degree of reproducibility of these data (11). A final conclusion from these data is warranted, namely that the degree and type of response initiated by ACTH administration is independent of "resting" levels of steroid excretion in normals. There appears to be an inherent capacity to respond to adrenal stimulation by increased steroid hormone production and this "capacity" to respond is not shared equally by all individuals. It may be that the "capacity" 
for response and not the usual level of adrenal function is the real delineator of the role of the adrenal gland in health and disease.

\section{SUM MARY}

The response of two young and two old men to continued ACTH administration has been studied by means of the extensive chemical and physical analytic techniques developed in this laboratory for isolation and quantitation of individual urinary steroids. This response was characterized by a prompt, sustained increase in urinary neutral steroids and the individual ketonic constituents of this group. This increase was greater relatively for those precursors and metabolites characterized by an 11-oxygen function, such as Kendall's compounds $E$ and F, their "tetrahydro" derivatives, 11 ketoetiocholanolone, 11-hydroxyandrosterone, and 11-hydroxyetiocholanolone.

The increase in 11-desoxy 17-ketosteroids was relatively less and due primarily to increased excretion of etiocholanolone; irregularly there were increases in androsterone excretion.

Although the response to ACTH may be characterized in general terms, there were striking qualitative and quantitative individual variations. From the four examples presented these variations appear little, if at all, related to chronologic age. These variations are attributed to the inherent biologic characteristics of the individual.

\section{ACKNOWLEDGMENTS}

We should like to express our deep appreciation to Dr. Nathan Shock and his group for allowing us joint participation in studies on these subjects, and for providing us with the results of the very extensive clinical studies they performed.

Metabolic balance data on these men have already been published elsewhere (4).

The authors wish to express their appreciation to the large group of devoted Research Assistants and techni- cians who made much of the work possible. The routine chemical and chromatographic separations were carried out by a group under the supervision of Madeleine Stokem and Ruth Jandorek. The colorimetric analyses were under the supervision of Olga Teager. The infrared spectrometry was under the supervision of Friederike Herling.

\section{REFERENCES}

1. Dobriner, K., Studies in steroid metabolism. XIX. The $\alpha$-ketosteroid excretion pattern in normal males. J. Clin. Invest., 1953, 32, 940.

2. Solomon, D. R., and Shock, N. W., Studies of adrenal cortical and anterior pituitary function in elderly men. J. Gerontol., 1950, 5, 302.

3. Pincus, G., Measures of stress responsivity in younger and older men. Psychosom. Med., 1950, 12, 225.

4. Duncan, L. E., Jr., Solomon, D. H., Rosenberg, E. K., Nichols, M. P., and Shock, N. W., The metabolic and hematologic effects of the chronic administration of ACTH to young and old men. J. Gerontol., 1952, 7, 351.

5. Dobriner, K., Lieberman, S., and Rhoads, C. P., Studies in steroid metabolism. I. Methods for the isolation and quantitative estimation of neutral steroids present in human urine. J. Biol. Chem., 1948, 172, 241.

6. Mason, H. L., Isolation of adrenal cortical hormones from urine: 17-hydroxycorticosterone and 17-hydroxy-11-dehydrocorticosterone. J. Biol. Chem., 1950, 182, 131.

7. Ronzoni, E., The excretion of dehydroisoandrosterone during adrenal stimulation with adrenocorticotropic hormone. J. Clin. Endocrinol. \& Metab., 1952, 12, 527.

8. Landau, R. L., Knowlton, K., Lugibihl, K., and Kenyon, A. T., The effect of adrenal stimulation with adrenocorticotrophic hormone on the excretion of dehydroisoandrosterone in man. Endocrinology, 1951, 48, 489.

9. Fukushima, D. K., Dobriner, K., and Gallagher, T. F., Metabolic studies with deuterium steroid hormones. Federation Proc., 1951, 10, 185.

10. Gallagher, T. F., Unpublished data.

11. Dobriner, K., Studies in steroid metabolism. XX. The reproducibility of the urinary steroid pattern in humans. J. Clin. Invest., 1953, 32, 950. 